\title{
Hydrophobic Gas-Diffusion Media for Polymer-Electrolyte Fuel Cells by Direct Fluorination
}

\author{
Trung Van Nguyen,,${ }^{a, *, z}$ Azita Ahosseini, ${ }^{a}$ Xuhai Wang, ${ }^{a}$ Venkata Yarlagadda,,${ }^{a, * *}$ \\ Anthony Kwong, ${ }^{\mathrm{b}}$ Adam Z. Weber, ${ }^{\mathrm{b}, * * *}$ Phengxay Deevanhxay, ${ }^{\mathrm{c}, \mathrm{d}}$ Shohji Tsushima, ${ }^{\mathrm{c}, * * *, \mathrm{e}}$ \\ and Shuichiro Hirai ${ }^{\mathrm{c}}$ \\ ${ }^{a}$ Department of Chemical \& Petroleum Engineering, The University of Kansas, Lawrence, Kansas 66045, USA \\ ${ }^{b}$ Lawrence Berkeley National Laboratory, Berkeley, California 94720, USA \\ ${ }^{c}$ Department of Mechanical and Control Engineering, Tokyo Institute of Technology, Ookayama, Tokyo, Japan
}

\begin{abstract}
A polymer-electrolyte fuel cell depends on proper water management to obtain high performance. During operation, liquid water is generated in the cell. When it is not properly and adequately removed, accumulation leads to poor fuel-cell performance by reducing and blocking the gas pores in the catalyst and gas-diffusion media. To address this problem, gas-diffusion media are often coated with a wet-proofing agent. This approach results in reduced pore size and volume resulting in lower transport properties, as well as inducing durability and performance issues due to the inherent non-uniformity. To overcome these issues, an alternative wet-proofing process called direct fluorination was developed. In this approach, fluorine gas reacts with carbon to create a more uniform, durable, and consistent wet-proof surface without affecting the morphology of the media. The fluorinated media showed capillary pressure properties that are more suitable for fuel-cell application. Fuel cells with fluorinated materials in the cathodes showed better performance, lower ohmic resistance, and lower liquid water amount in the cathode. These advantages are attributed to having a better wet-proofed fluorinated media at the cathode that forces water back to the anode, thereby keeping the membrane more hydrated and reducing the amount of water in and transported out of the cathode.

(c) The Author(s) 2015. Published by ECS. This is an open access article distributed under the terms of the Creative Commons Attribution 4.0 License (CC BY, http://creativecommons.org/licenses/by/4.0/), which permits unrestricted reuse of the work in any medium, provided the original work is properly cited. [DOI: 10.1149/2.0411514jes] All rights reserved.
\end{abstract}

Manuscript submitted August 10, 2015; revised manuscript received September 21, 2015. Published October 9, 2015. This was Paper 1628 presented at the Honolulu, Hawaii, Meeting of the Society, October 7-12, 2012.

A proton-exchange-membrane (PEM) fuel cell depends on proper water management to obtain high power density and energy efficiency. During operation, liquid water is generated in the fuel cell at the cathode due to oxygen reduction, and may also exist at the anode due to transport through the membrane or condensation. When it is not properly and adequately removed, this liquid-water accumulation leads to poor fuel-cell performance by blocking the gas pores in the catalyst and gas-diffusion media (GDM) and forming an additional transport barrier over the reactive area. To address this problem, GDM, such as non-woven carbon papers or woven carbon cloths, used in PEM fuel cell electrodes are treated with polytetrafluoroethylene (PTFE) or tetrafluoroethylene-hexafluoropropylene (FEP) to wet-proof the substrate surface. Wet-proofed surface in the GDM repels liquid water and provides pathways for gaseous reactant transport. ${ }^{1-4}$ Furthermore, when bilayer GDM that have a dense and hydrophobic microporous layer (MPL) supported by a macroporous layer are used in a PEM fuel cell, it is believed that lower liquid-water-saturation levels in the cathode are achieved because the dense and highly hydrophobic MPL 1) reduces the liquid-water saturation level at the catalyst layer/MPL interface thus enabling gas access from the GDM to the catalyst layer, 2) reduces the amount and locations of liquid water that can be transported from the catalyst layer into the macroporous support layer, and 3) forces a significant amount of liquid water in the cathode across the membrane back to the anode, thereby helping to make the anode side of the membrane more hydrated. ${ }^{5-6}$ In addition to the effect on water management, the dense and smooth MPL also provides better electrical contact between the catalyst layer and the GDM. The use of wet-proofed single and, especially, bilayer GDM has resulted in significantly improved fuel-cell performance. ${ }^{1-13}$

GDM are currently wet-proofed by physical coating of the media substrate with either PTFE or FEP. The physical coating process is discussed in detail in the literature ${ }^{7-10}$ and can be summarized as

\footnotetext{
*Electrochemical Society Fellow.

**Electrochemical Society Student Member

****Electrochemical Society Active Member.

${ }^{\mathrm{d}}$ Present Address: Department of Chemistry, National University of Laos, Vientiane, Laos.

Present Address: Department of Mechanical Engineering, Osaka University, Osaka, Japan.

${ }^{\mathrm{z} E-m a i l: c p t v n @ k u . e d u}$
}

follows. For the macroporous GDM, the process involves washing the GDM in a solvent to clean the surface followed by repeated dipping of the GDM in a diluted solution of PTFE or FEP and drying until a desired solid loading is achieved. To create a MPL, carbon powder and PTFE dispersed in a solution are mixed, applied onto one side of the macroporous layer and heat treated to remove the solvent and disperse the PTFE.

It has been found that PEM fuel cells with PTFE-coated GDM in general have greater performance than those with untreated GDM, and the best performance was obtained when the PTFE contents are optimal, $\sim 10 \%$ by weight for the macroporous substrate layer and $\sim 20$ to $35 \%$ for the MPL depending on the operating conditions. ${ }^{3,7,10}$ Prior studies also showed that too little or too much PTFE contents in the GDM can lead to poor PEM fuel-cell performance. For example, low PTFE content results in high liquid-water saturation level in the porous structure and pore flooding during operation, while high PTFE content can lead to reduced pore volume and size and low gas permeability. High PTFE content can also result in high ohmic resistance in the MPL and high contact resistance for the GDM when the PTFE accumulates on the GDM surfaces.

These effects can be explained further as follows. As more PTFE is added to a GDM, more surfaces of the fibers, for the case of the macroporous layer, and carbon powder, for the case of the MPL, in the GDM are covered with PTFE, which increases the hydrophobicity of the GDM, reduces the liquid-water saturation level and enhances liquid-water removal rate in the GDM during operation. However, once the surface of a particular region in the GDM is fully covered, additional PTFE leads to reduced pore volume and size, and higher contact resistance if the excessive PTFE resides on the outer surface of the GDM. For the MPL, excessive PTFE content can lead to both high contact and ohmic resistances because the PTFE can form a layer between the carbon particles as compared to the macroporous layer where the carbon fiber network is already established and the PTFE can only coat the fiber surface. Cheng et al. showed that although open pores in the GDM were observed in the un-treated GDM, most of these pores were sealed when the PTFE content was over $40 \%$ by weight. ${ }^{14}$ Furthermore, because of the interconnecting carbon-fiber-network nature of a GDM like the macroporous substrate layer, more PTFE accumulates at the intersections of the carbon fibers than on the fibers leading to nonuniform distribution of PTFE. ${ }^{15}$ So, complete coverage 


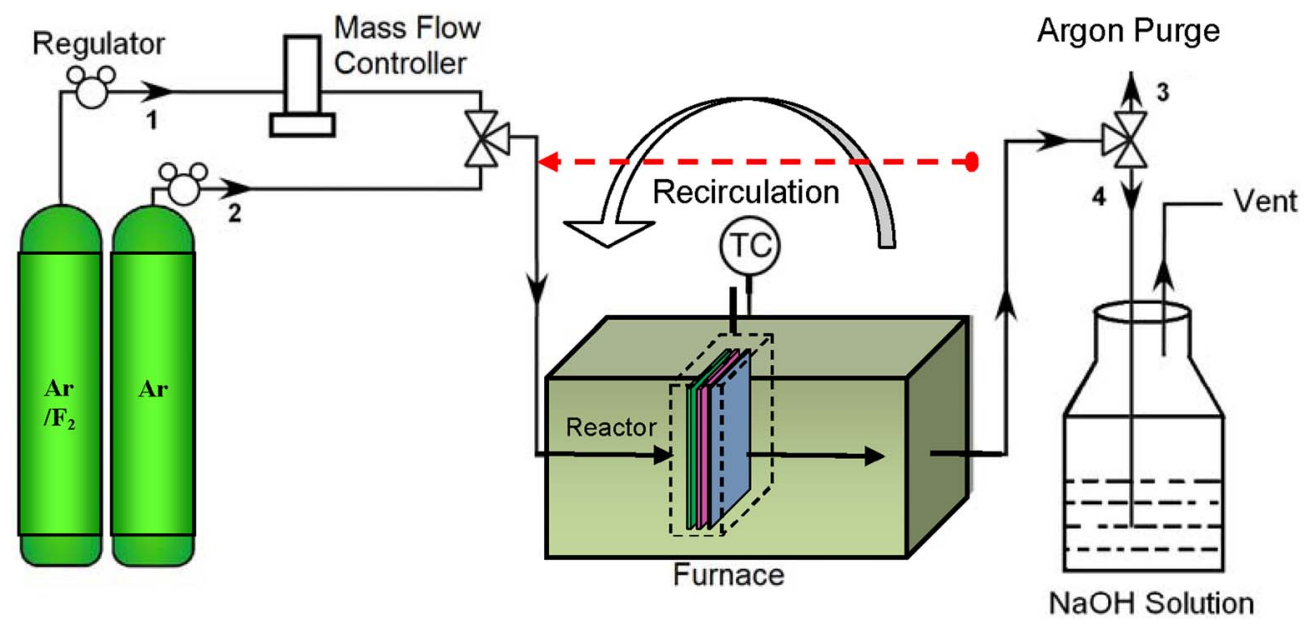

Figure 1. Experimental setup used for direct fluorination of gas diffusion media.

of the fibers surface requires excessive PTFE content and significant pore volume and size reduction in a GDM. Prior work by Wang et al. ${ }^{16}$ also showed that PTFE often migrates to the outer surface of the GDM during high temperature treatment resulting in both nonuniform distribution of PTFE in the pores and excessive PTFE on the outer surface of the GDM. To avoid this issue, a low PTFE content is used in existing GDM in PEM fuel cells with the consequential effect of only partial PTFE coverage of the carbon fibers. The GDM wet-proofed by physical coating with a wet-proof agent like PTFE or FEP also exhibit durability issues. Since the PTFE coating is attached to the substrate by means of weak Van der Waals forces, it could separate from the substrate surface due to physical changes like expansion and contraction, water penetration into the PTFE and carbon interface, and shear force by fluid flow during the operation of a fuel cell. ${ }^{15}$

To address the issues highlighted above, a surface modification process called "direct fluorination" was investigated to see if it could be used to create more uniform and durable wet-proofed surface in a GDM with minimal or no effect on its morphological and transport properties. ${ }^{17}$ The surface modification process by direct fluorination involves reacting carbon-based GDM with fluorine gas to form functional groups such as $\mathrm{CF}, \mathrm{CF}_{2}$, and $\mathrm{CF}_{3}$ on the surface of the carbon substrate to create the surface hydrophobicity. The bulk carbon material is not affected by this method. ${ }^{18-21}$ Direct fluorination at high temperatures is chosen because of the high surface coating level that can be attainable with this approach and the fact that only the outer surface of the substrate was modified while the inner structure is unchanged. Also, this process is simpler and the potential for contamination by another component is eliminated. Since the wet-proof surface is formed by fluorine atoms that are chemically bonded to the carbon substrate, it is expected to be much more durable. Also, since the thickness of this surface layer is at the nanometer scale, it is not expected to affect the void volume or gas permeability of the porous medium. A somewhat related concept was investigated by Thomas et al. using electrografting of hydrophobic molecules onto the carbon-fiber surface. ${ }^{22}$ The electrografting approach offers the use of different wet-proofing molecules and low-temperature synthesis. However, this process can potentially introduce contaminants into the GDM, and unless one can ensure that all of the carbon surface is in contact with the electrografting electrolyte, complete coverage of the carbon surface may not be achieved.

Direct fluorination of carbon materials has been investigated for many applications ranging from low friction and durable materials for lubrication to materials for lithium-ion batteries. ${ }^{18-31}$ In these studies, carbon powder materials were reacted with fluorine gas from 350 to $600^{\circ} \mathrm{C}$, with the value of $\mathrm{x}$ in the compound $\mathrm{CF}_{\mathrm{x}}$ on the surface of the carbon materials varying from 0.6 up to 1 , respectively. Treatment at lower temperatures $\left(<300^{\circ} \mathrm{C}\right)$ was found to result in materials with poorly fluorinated surfaces, and treatment at higher temperatures $\left(<700^{\circ} \mathrm{C}\right)$ resulted in materials with higher $\mathrm{x}$ values due to the formation of $\mathrm{CF}_{2}$ and $\mathrm{CF}_{3}$ groups on the surface and loss in carbon material due to the formation of gaseous $\mathrm{CF}_{4}$ product.

In this paper, the direct-fluorination process used to wet-proof carbon GDM is discussed and the characterization and fuel-cell performance test results are presented.

\section{Experimental}

Direct fluorination. - The surface modification process by direct fluorination involves reacting carbon-based GDM with fluorine gas to form functional groups such as $\mathrm{CF}, \mathrm{CF}_{2}$, and $\mathrm{CF}_{3}$ on the surface of the carbon substrate to create surface hydrophobicity. A process flow diagram of the experimental setup is shown in Figure 1. The reaction was carried out in a small reactor placed in a temperature controlled oven (Carbolite, Model ELF 11/6B). The reactor and the tubing and fittings attached to the reactor were made of nickel, which was selected because of its corrosion resistance to fluorine at high temperature. The reactor consisted of two nickel plates with a single through-hole drilled on each plate for gas inlet and outlet. The plates were sealed by a rectangular ring graphite gasket with the cut-out center $(3.4 \mathrm{~cm} \times 3.4 \mathrm{~cm})$ opening for the samples and gas flow. A thermocouple embedded in one of the end plates was used to monitor the temperature of the reactor during the treatment process. In the preliminary study, flat graphite foil (GrafTech, GTA-0.015 inch) samples, that were slightly smaller than the opening of the graphite gaskets, were used to determine the optimal treatment temperatures (i.e., temperatures that would lead to highest water contact angles). Once an optimal temperature range was determined from the solid graphite sheet study, porous GDM were used to determine the optimal treatment time. When GDM samples were used, the thickness of the graphite foil gaskets was selected such that the GDM sample was slightly compressed around the edges to force the fluorine gas mixture to flow through the sample.

Figure 2 shows a cross-sectional view of the reactor and the placement of the sample(s) for two different configurations. The 1-sample configuration was used to prepare samples for physical testing (contact angle, capillary pressure, SEM, XPS, and EDX). The 3-sample configuration was used to prepare samples (the middle layer) for fuel-cell testing. In the 3-sample arrangement, the outer surfaces of the middle layer that was used for fuel-cell testing were protected from being fluorinated so that good electrical contact of the GDM with the catalyst layer and the flow-field plate was maintained. Note that multiple middle layers could be used to treat multiple samples simultaneously; more on this configuration will be discussed later in the results section. To protect the external carbon surface of the wanted GDM from 


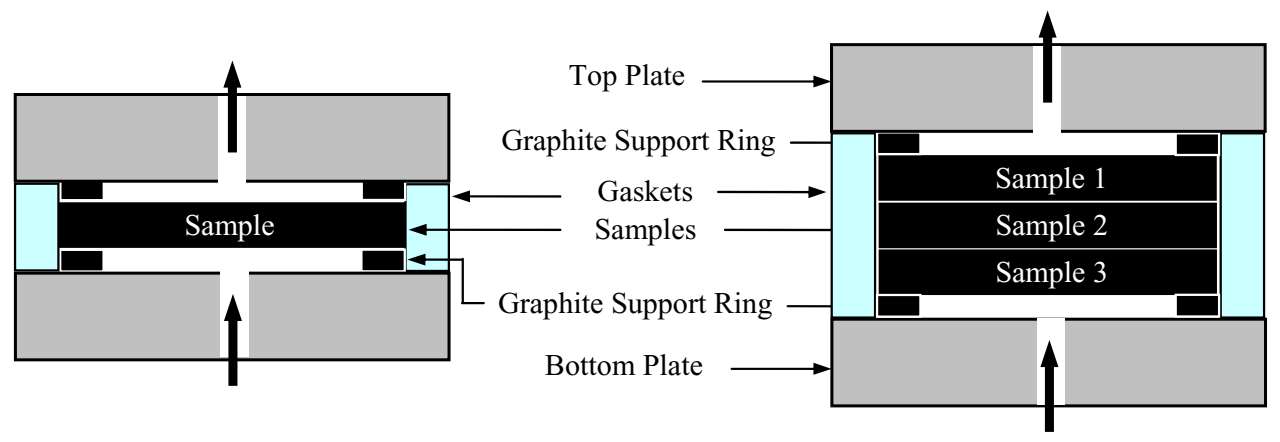

Figure 2. Cross-sectional view of reactor used for direct fluorination of carbon GDM, (a) single-layer configuration and (b) multiple-layer configuration.

fluorination, the GDM were stacked with similar surfaces in contact. That is, the MPL of one GDM was in contact the MPL of another GDM, and the GDL of one GDM was in contact with the GDL of another GDM. A premixed gas mixture of $20 \%$ fluorine in argon (Linde Specialty Gases) was used for the treatment. Gas mixture with higher fluorine compositions could be used and might reduce the reaction time; however, for safety, a lower fluorine composition gas mixture was chosen. Furthermore, to minimize the emission of unreacted fluorine, recirculation of the fluorine-gas mixture was also applied (see Figure 1). Pure argon was used for purging at the beginning and end of the experiment. A solution of $1 \mathrm{M} \mathrm{NaOH}$ (or $\mathrm{KOH}$ ) was used to neutralize unreacted fluorine $\left(2 \mathrm{~F}_{2}+4 \mathrm{NaOH} \rightarrow 4 \mathrm{NaF}+2 \mathrm{H}_{2} \mathrm{O}+\mathrm{O}_{2}\right)$ in the exhaust stream. The whole setup was checked for gas leaks prior to each test and operated in a vented hood.

The operating procedure was as follows:

1. The reactor was continuously purged with argon, set at $120 \mathrm{kPa}$, as the oven was heated to the operating temperature. The temperature of the reactor was monitored with an attached thermocouple. During this time, the gas exhaust was vented directly to the hood.

2. Once the reactor reached the desired temperature, the setup was again checked for gas leaks. Once no leak was found, gas flow was switched from pure argon to the fluorine and argon gas mixture, set between 115 to $120 \mathrm{kPa}$, and the exhaust stream was switched from direct vent to the $\mathrm{NaOH}$ treatment bottle and vented thereafter to the hood. Once the reactor was considered to be sufficiently purged of argon, the fluorine-argon gas exhaust could be shut off and the system could be operated under constant pressure batch or closed-loop mode to minimize emission of unreacted fluorine.

3. The GDM was treated for a specified duration and the gas flow was then switched back to pure argon as the reactor was allowed to cool to room temperature.

4. Once the temperature of the reactor reached room temperature all gases were shut off and the reactor was removed from the oven for disassembly and sample removal.

5. The materials were stored in ambient environment and used without additional treatment.

Physical and chemical characterization.- XPS and SEM were used to determine the surface and bulk C:F ratio and morphology of graphite foil and GDM after treatment. Confirmation of fluorination of interior surface was done by measuring XPS and SEM/EDX of crushed GDM. Static contact angle of water droplet on the media's solid surface was measured for both the graphite foil and GDM to determine the relative change in the hydrophobicity of the material's external surface. The water-droplet contact angles were obtained using the procedure described in the appendix. Multiple droplets ( 3 to 4 ) on various locations on the sample were used to check for uniformity. The reported values are the averaged values of these measurements. Both surfaces of single-layer GDM (i.e., only GDL) were analyzed. For bilayer GDM, only the exposed surface of the macroporous GDL was analyzed. For internal surfaces, capillary pressure curves of untreated versus treated GDM were measured using the water-intrusion method reported in the literature. ${ }^{32,33}$

Fuel-cell testing. - The fuel-cell performance of fluorinated samples was evaluated at the University of Kansas in a $\mathrm{H}_{2}$ /Air PEM fuel cell at room temperature $\left(\sim 22^{\circ} \mathrm{C}\right)$ to assess the effect of these materials on the performance of a PEM fuel cell as compared to that obtained with conventionally treated commercial GDM. These fluorinated materials were also tested at Tokyo Institute of Technology (Tokyo Tech) in a PEM fuel cell set up that was equipped with a soft $\mathrm{X}$-Ray radiography system to allow in-situ monitoring of the liquid water dynamics in the electrode membrane assembly (MEA) of a PEM fuel cell during operation. This setup was used to gain more insights of the relationship between the liquid-water dynamics (saturation level and distribution) in the various layers in an MEA and the fuel-cell electrical performance.

For the data obtained at the University of Kansas, we used a fuel cell that had an active area of $2.25 \mathrm{~cm}^{2}(1.5 \mathrm{~cm}$ by $1.5 \mathrm{~cm})$, an anode made of a conventionally treated commercial bilayer GDM (SGL $35 \mathrm{BC}$ ) with a $0.5 \mathrm{mg} \mathrm{Pt} / \mathrm{cm}^{2}$ loading catalyst layer applied on the MPL side, a cathode made of a fluorinated bilayer GDM (SGL 30 $\mathrm{AC}$ or $35 \mathrm{BC}$ ) with a $0.5 \mathrm{mg} \mathrm{Pt} / \mathrm{cm}^{2}$ catalyst layer applied on the MPL side, and a Nafion 212 membrane. Interdigitated flow fields were used on both anode and cathode sides. Solid PTFE gaskets with proper thicknesses were used to ensure that GDM were compressed to only $80 \%$ of their uncompressed thicknesses. This approach prevents the GDM from being crushed, eliminates the need to control the compression level, and is more reproducible. For the baseline case, the same electrode used for the anode was also used for the cathode. Hydrogen gas saturated with water vapor at $22^{\circ} \mathrm{C}$ and $120 \mathrm{kPa}$ was circulated through the anode $\left(\mathrm{H}_{2}\right)$ side in a closed loop at $660 \mathrm{~cm}^{3} / \mathrm{min}$ or $42 \mathrm{~A} / \mathrm{cm}^{2}$ equivalent, and air at ambient pressure and $66 \mathrm{~cm}^{3} / \mathrm{min}$ or $1.8 \mathrm{~A} / \mathrm{cm}^{2}$ equivalent was applied to the cathode $\left(\mathrm{O}_{2} /\right.$ air $)$ side.

For the data obtained at Tokyo Tech, we used a specially designed fuel cell and setup, as shown in Figure 3, to allow the liquid-water distribution in the fuel cell to be imaged by soft X-ray during operation. More details on this setup are available in an earlier work. ${ }^{34}$ This fuel cell had an active area of $0.12 \mathrm{~cm}^{2}(0.1 \mathrm{~cm}$ by $1.2 \mathrm{~cm})$, an anode made of a conventionally treated commercial bilayer GDM (SGL 24 BC) with $0.27 \mathrm{mg} \mathrm{Pt} / \mathrm{cm}^{2}$ catalyst layer applied on the MPL side, a cathode made of a fluorinated bilayer GDM (SGL 30 AC) with 0.27 $\mathrm{mg} \mathrm{Pt} / \mathrm{cm}^{2}$ catalyst layer applied to the MPL side, and a Nafion 212 membrane. Hydrogen and air saturated with water vapor at $65^{\circ} \mathrm{C}$ and $50 \mathrm{~cm}^{3} / \mathrm{min}\left(60 \mathrm{~A} / \mathrm{cm}^{2}\right.$ equivalent for $\mathrm{H}_{2}$ and $25 \mathrm{~A} / \mathrm{cm}^{2}$ for air) flow rate were applied to the anode and cathode, respectively, while the fuel cell was maintained at $65^{\circ} \mathrm{C}$. A fuel cell test system by NF Corporation comprising of Fuel Cell Analyzer (AS-510-4), Frequency Response Analyzer (FRA 5014), and Electrochemical Analyzer (AS-510-ECA03) was used to obtain the fuel cell polarization curves. The fuel-cell internal resistance was acquired at OCV and during load operation at $10 \mathrm{kHz}$ using the same system. Liquid-water content profiles in the fuel cell were obtained by soft X-ray radiography using the procedure given in an earlier work. ${ }^{34}$ Table I summarizes the 
(b)

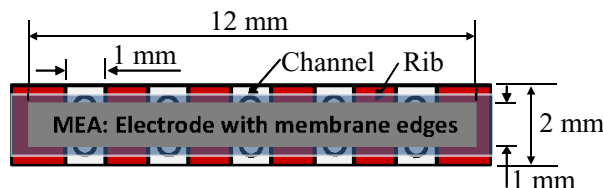

(c)

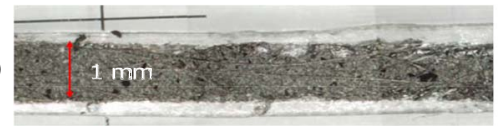

(a)

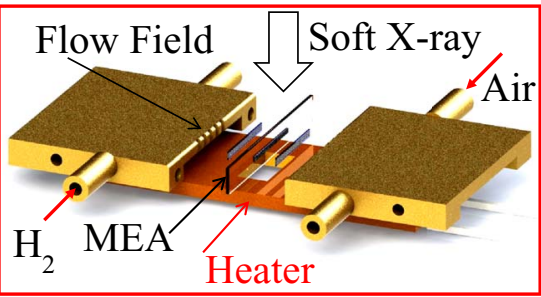

Figure 3. Schematic of fuel cell setup for soft X-ray radiography (a), flow field-MEA configuration - top view (b), MEA - top view (c).

Table I. GDM properties relevant to the fuel-cell test.

\begin{tabular}{lcccc} 
& $\begin{array}{c}\text { Thickness } \\
(\mu \mathrm{m})\end{array}$ & Porosity & $\begin{array}{c}\text { Areal Weight } \\
\left(\mathrm{g} / \mathrm{m}^{2}\right)\end{array}$ & $\begin{array}{c}\text { Air Permeability } \\
\left(\mathrm{cm}^{3} / \mathrm{cm}^{2}-\mathrm{s}\right)\end{array}$ \\
\hline SGL 24BA & 190 & 84 & 54 & 60 \\
SGL 24BC & 235 & 76 & 100 & 0.6 \\
SGL 30AA & 300 & 83 & 90 & 43 \\
SGL 30AC* & 330 & 76 & 140 & 0.56 \\
SGL 35BA & 300 & 90 & 54 & 170 \\
SGL 35BC* & 325 & 81 & 110 & 1.5
\end{tabular}

*With microporous layer

Source: SGL Carbon product brochure

relevant properties of the GDM used in the fuel-cell studies. Multiple types were used because the supplier (SGL Carbon) stopped manufacturing some of the materials during the course of this study, and some materials were selected because of their applicability in different applications including transportation and portable, air-breathing PEM fuel cells.

\section{Results and Discussion}

Direct fluorination.- Since the static liquid-water-droplet contact-angle technique was used to characterize the surface hy- drophobicity created by the direct fluorination process, solid graphite sheets were used initially to minimize errors introduced by droplet pinning on discontinuous substrates. ${ }^{35}$ Once the optimal temperature range was determined with these graphite solid sheets, these same conditions were applied to the porous carbon materials. Figure 4 shows both the preliminary results obtained with solid graphite sheets and those with porous carbon-fiber media. A reaction time of 10 minutes was found to be more than adequate to fluorinate the exposed surfaces of a $3 \mathrm{~cm}$ by $3 \mathrm{~cm}$ solid graphite sheet. The contact-angle results for the solid graphite sheet study are shown as red square symbols in Figure 4. Note that the contact angles given at $22^{\circ} \mathrm{C}$ were for untreated samples, included here for comparison purpose. The results for this case study show that the sample surface hydrophobicity increased with the treatment temperature and reached its highest levels in the temperature range of 350 to $408^{\circ} \mathrm{C}$. Beyond this range, the surface hydrophobicity decreased, and, as suggested in the previous section, this decrease in surface hydrophobicity could be attributed to the formation of volatile $\mathrm{CF}_{4}$ product exposing the carbon sublayers and making the surface less fluorinated. Note that the surface contact angle of the sample treated at $500^{\circ} \mathrm{C}$ was very close to that of the untreated sample $\left(67^{\circ}\right.$ vs. $\left.62^{\circ}\right)$. Finally, the amount of carbon loss by the formation of volatile fluorocarbon products was very small as no weight change was detectable by the balance (accurate to $10 \mu \mathrm{g}$ while sample size was $>90 \mathrm{mg}$ ) used in this study.

The blue diamond symbols represent the contact angle results of a GDM (Toray 060) treated at the same temperature range and for the

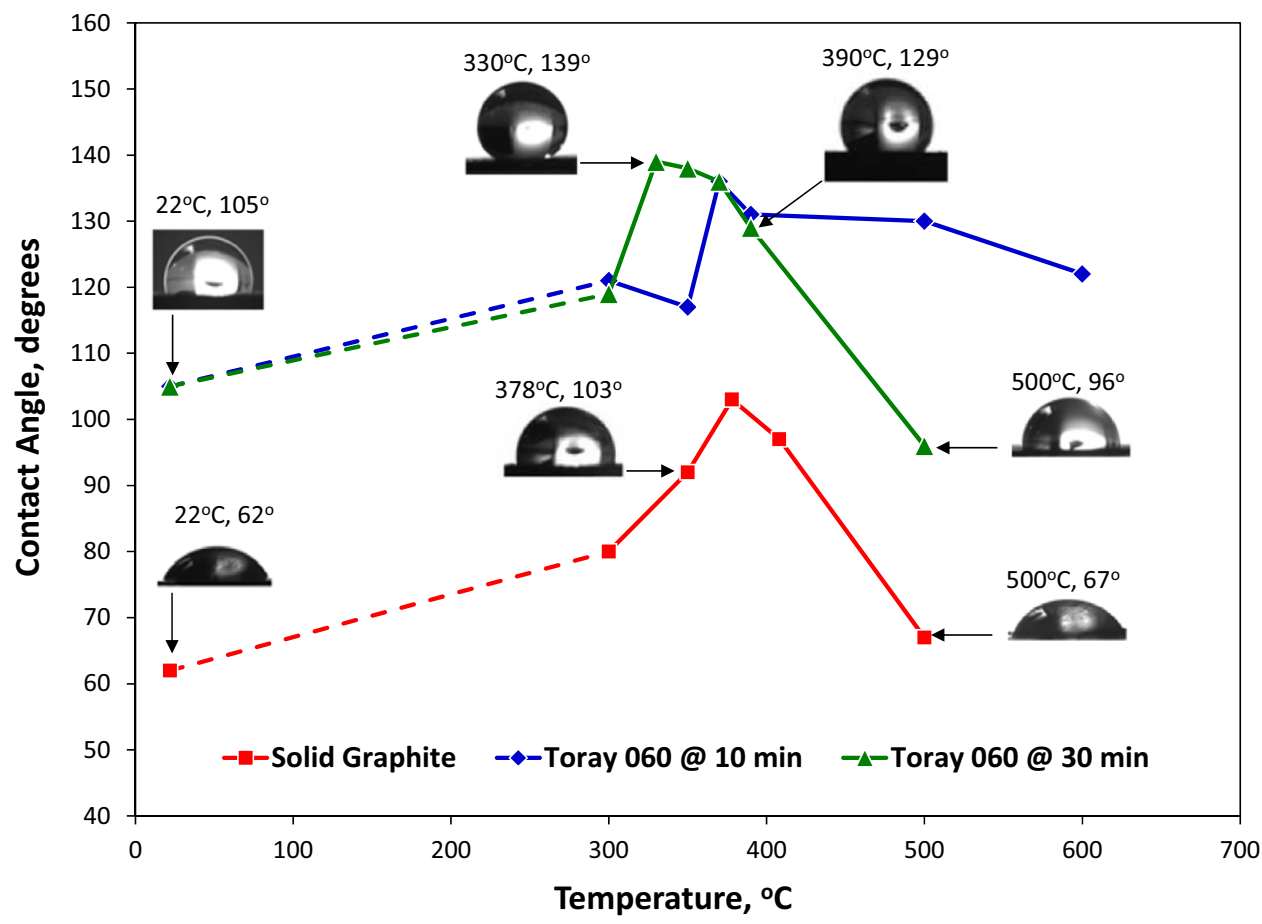

Figure 4. Contact angle results of fluorinated solid graphite sheets and porous carbon GDM (Toray 060). 


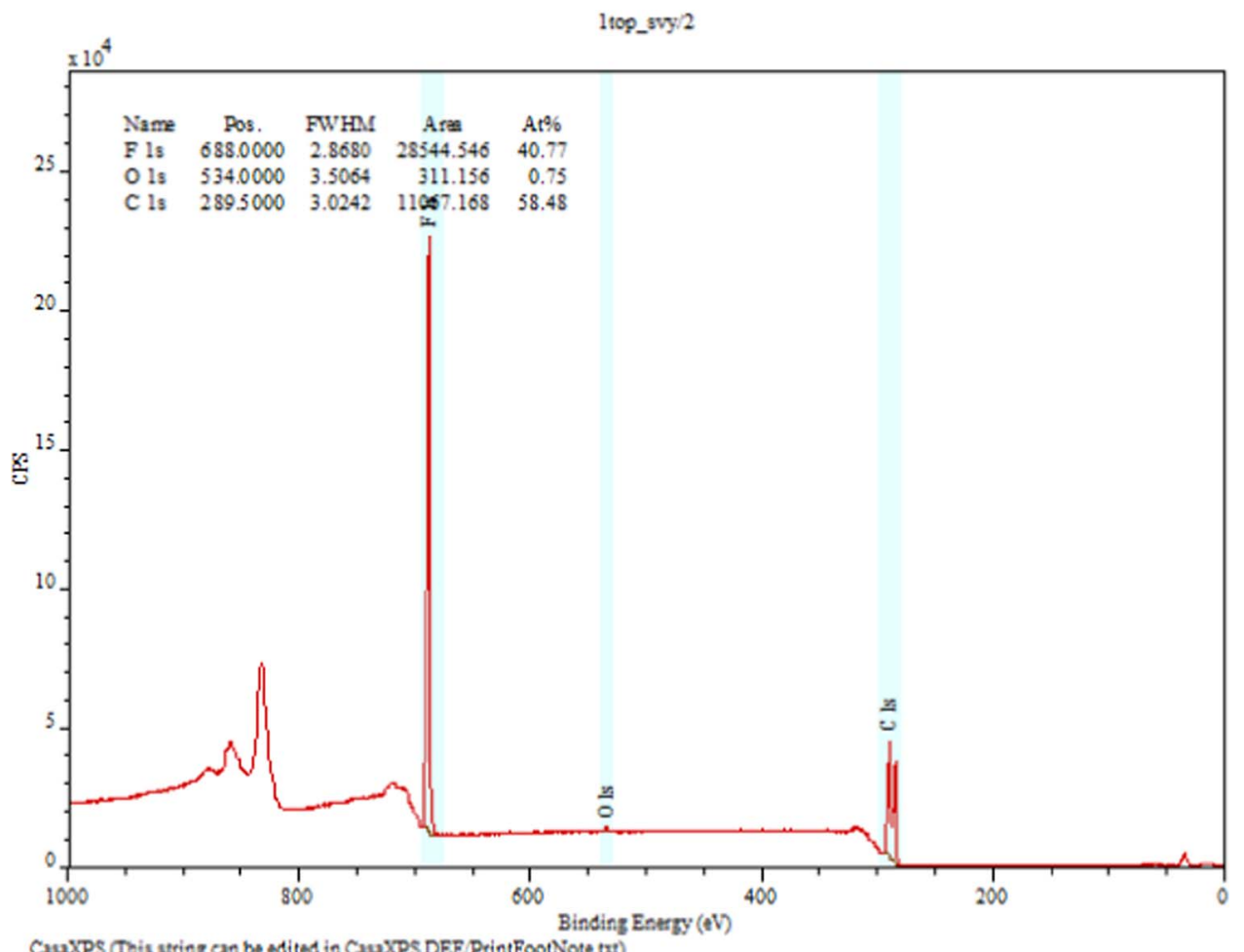

Figure 5. XPS result of fluorinated Toray 060 at $350^{\circ} \mathrm{C}$ for 30 minutes showing fluorine to carbon ratio on surface of $41: 58$ or close to 1:1 for C-F formation.

same duration (10 minutes), and the green triangle symbols are those of the same GDM (Toray 060) treated for 30 minutes. First, the results of these runs show that porous media with rougher and non-continuous surface show higher static water-droplet contact angles than flat, solid graphite sheets. Second, the shape of the contact angle results in the 350 to $600^{\circ} \mathrm{C}$ range for the GDM treated for 10 minutes is different from that of the GDM treated for 30 minutes; however, the shape of the results of the GDM treated for 30 minutes is very similar to that of solid graphite sheets treated for 10 minutes. This difference shows that for high carbon surface area materials like Toray 060, longer reaction time was needed to fluorinate all the exposed carbon surface area. Finally, for bi-layer GDM that have even higher surface areas (e.g., MPLs composed of fine carbon powder), a longer (1 hour) treatment time was used.

In general, higher variations in contact angle were observed with porous GDM than with solid graphite sheets as expected because of the non-uniform surface roughness of porous GDM. XPS and EDX results of crushed GDM confirmed that fluorination occurred across the sample. However, complete fluorine coverage could not be ascertained because of exposed cross-sections of broken carbon fibers. The capillary-pressure curves, presented later, are reliable indicators of internal wetting properties of GDM and provide better evidence of fluorination of the internal GDM carbon surfaces. Limited durability was also evaluated by comparing the liquid-water-droplet contact angle on the sample before and after boiling the sample in DI water for $2 \mathrm{hr}$ and drying it overnight at $80^{\circ} \mathrm{C}$. The averaged contact angle after boiling decreased by 3 to 5 degrees. This level of contact-angle decrease was also observed for PTFE treated GDM; so no conclusion was drawn. The decrease could be due to contamination or small changes in the carbon-fiber structure during boiling. While this test may be too short to determine the long-term durability, fluorinated carbon developed for other applications, such as lubrication, has shown high durability. Finally, note that water-droplet contact angles, XPS, and SEM/EDX were used as qualitative data to optimize the direct-fluorination process. The results of the capillary-pressure measurements and fuel-cell test were used to correlate quantitatively the effect of wet-proofing by fluorination.

Physical and chemical characterization.- No visible difference in appearance was observed; the SEM/EDX results for a GDM (Toray 060) fluorinated at $350^{\circ} \mathrm{C}$ for 30 minutes shows that the amount of fluorine needed to wet-proof these carbon GDM was extremely small. Theoretical coverage of a GDM like Toray 060 with $0.4 \mathrm{~m}^{2} / \mathrm{g}$ of BET area with fluorine would require about $0.7 \mathrm{mg}$ of fluorine per $\mathrm{g}$ of GDM (for C:F of 1:1) or $0.07 \mathrm{wt} \%$, versus $10 \mathrm{wt} \%$ or higher for PTFE treated GDM used in PEMFCs. The XPS results for the

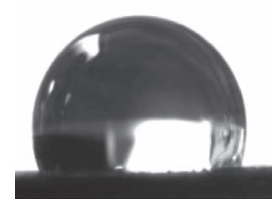

(a)

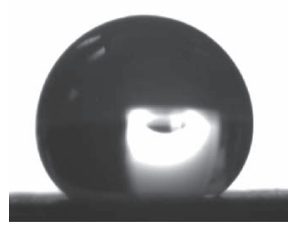

(b)

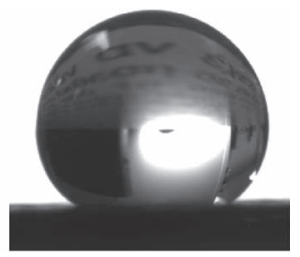

(c)

Untreated GDM $\left(115^{\circ}\right) \quad 10 \%$ PTFE wet proof GDM $\left(133^{\circ}\right) \quad$ Fluorinated GDM $\left(138^{\circ}\right)$

Figure 6. Surface wetting property of untreated and treated GDM (Toray 060) by conventional PTFE treatment and direct fluorination. 

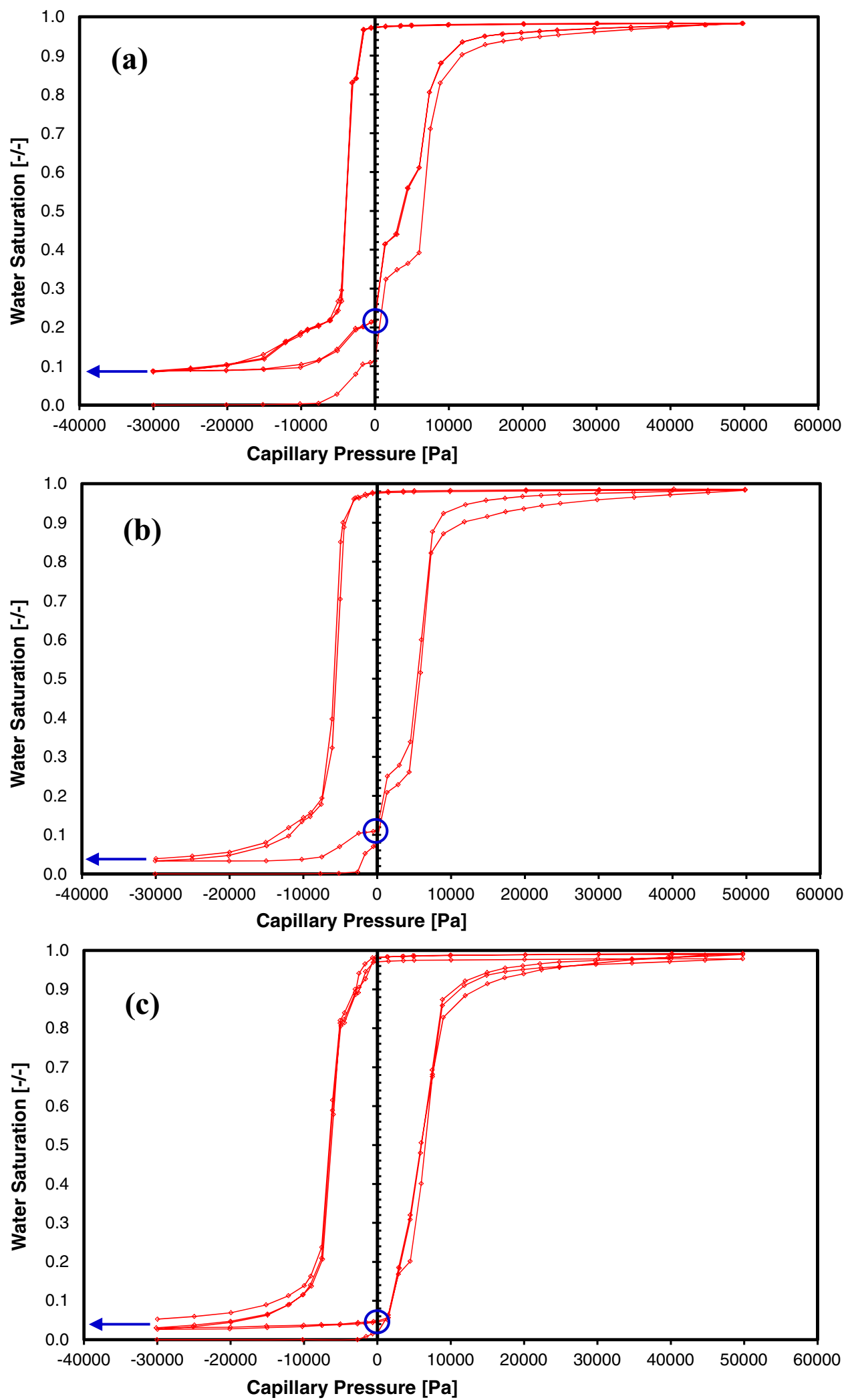

Figure 7. Capillary pressure curves of Toray 060 untreated (a), conventionally treated at $10 \mathrm{wt} \%$ PTFE (b), and fluorinated at $350^{\circ} \mathrm{C}$ and for $30 \mathrm{minutes}$ (c).

same fluorinated material (Figure 5) shows that the surface fluorine to carbon ratio is about $0.7: 1$ (41:58), which is very close to $1: 1$ for $\mathrm{C}-\mathrm{F}$ formation taking into consideration that XPS probes more than the first atomic layer of the surface. However, analysis of the XPS spectrum in the $\mathrm{C} 1 \mathrm{~s}$ region shows two carbon-fluorine peaks, $289 \mathrm{eV}$ for $\mathrm{CF}$ and $291 \mathrm{eV}$ for $\mathrm{CF}_{2}$. No further efforts were conducted to determine the ratio of $\mathrm{CF}$ to $\mathrm{CF}_{2}$ bonds.

Figure 6 shows the static water-droplet contact-angle results of Toray 060 untreated (a), commercial Toray 060 with 10 wt\% PTFE (b), and directly fluorinated Toray 060 at $350^{\circ} \mathrm{C}$ and 30 minutes (c). 


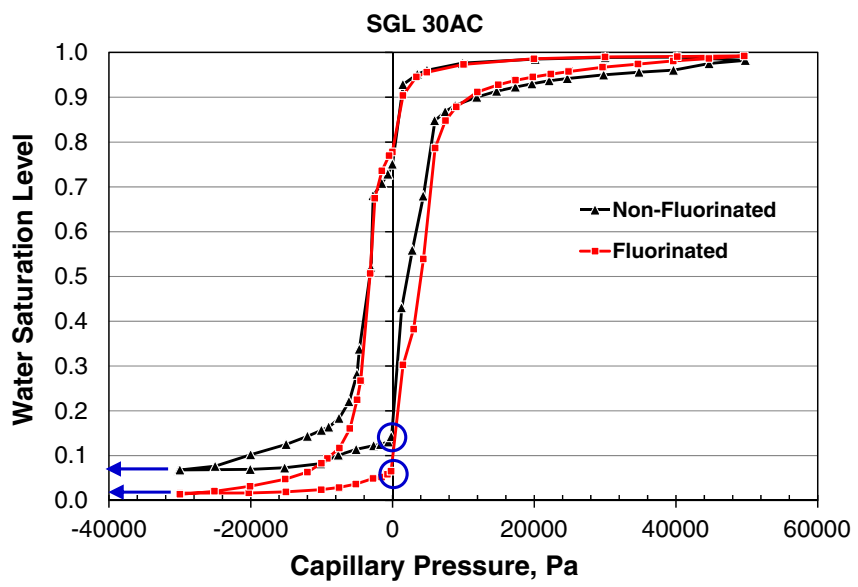

Figure 8. Capillary pressure curves of the macroporous part of a bilayer GDM (SGL $30 \mathrm{AC}$ ) as-received and after treatment by direct fluorination.

The results show that the untreated sample had the lowest contact angle $\left(115^{\circ}\right)$ and the treated samples had higher contact angles with that of the fluorinated sample slightly higher $\left(138^{\circ}\right)$ than that of the commercial material with $10 \mathrm{wt} \% \operatorname{PTFE}\left(133^{\circ}\right)$. It is important to highlight that conventionally treated commercial GDM tend to have higher PTFE on the outer surfaces than inside the materials. ${ }^{16}$

Figure 7 shows the capillary pressure $\left(\mathrm{P}_{\mathrm{C}}=\mathrm{P}_{\mathrm{L}}-\mathrm{P}_{\mathrm{G}}\right)-$ saturation curves for the same three Toray 060 samples. Each graph shows three cycles that started with a dry sample under negative capillary pressure (high gas pressure), where water was first imbibed and then cycled by changing the gas pressure. The most significant characteristics of these curves as far as fuel-cell application is concerned are the points highlighted by the blue circles and arrows. The liquid-water saturation levels marked by the arrows represent the irreducible saturation levels (i.e., the lowest water-saturation levels $\left(S_{\min }\right)$ or contents that one can reduce to in these GDM), where the liquid phase becomes discontinuous. These $S_{\min }$ levels would not be observed in an operating PEM fuel cell because PEM fuel cells don't operate in the negative capillary pressure region. The liquid-water saturation levels marked by the circles at zero capillary pressure, $\mathrm{P}_{\mathrm{L}}=\mathrm{P}_{\mathrm{G}},\left(\mathrm{S}_{\mathrm{Pc} 0}\right)$ are the lowest liquidwater saturation levels in these GDM that one can reduce to in a PEM fuel cell assuming saturated feeds and pressure equilibrium. These values are also very close to the breakthrough pressure of the liquid phase in the GDM, as witnessed by the sharp increase in the curves. ${ }^{5,6,36}$

As expected, the non-wet-proofed or untreated GDM had the highest $S_{\min }$ and highest liquid-water saturation level at zero capillary pressure $\left(\mathrm{S}_{\mathrm{Pc} 0}\right)$, demonstrating its greater hydrophilicity. With higher hydrophilicity or more wetting pore surfaces, more liquid water was trapped in the porous medium when the liquid water became disconnected during draining resulting in a higher $\mathrm{S}_{\min }$ level. The $10 \%$ PTFE Toray 060 and the directly fluorinated Toray 060 had the same $S_{\min }$ level, that was lower than that of the non-wet-proofed GDM, but the fluorinated GDM has the lowest $\mathrm{S}_{\mathrm{Pc} 0}$ level. This difference can be explained as follows. When the carbon-fiber surfaces were sufficiently wet-proof, the amount of trapped liquid water was sufficiently reduced to give the same $S_{\min }$ level, within measurement limits, during drainage. Note that because of the different pore sizes and pore interconnectivity, even a completely hydrophobic porous medium could still retain liquid water during drainage because of phase disconnection. The difference between the $S_{\min }$ level and the $\mathrm{S}_{\mathrm{P} \mathrm{c} 0}$ level, therefore, represents the small amount of trapped or hydrophilic pore volume remaining in the porous medium between those two capillary pressure points. In this case, we see that the difference between the $\mathrm{S}_{\mathrm{Pc} 0}$ and $\mathrm{S}_{\min }$ levels of the fluorinated sample was almost zero $\left(S_{\mathrm{Pc} 0}=S_{\min }=0.045\right)$ because the carbon surface was totally covered while that for the PTFE treated sample is $0.06\left(\mathrm{~S}_{\mathrm{Pc} 0}=0.105\right.$ and $\left.\mathrm{S}_{\min }=0.045\right)$.

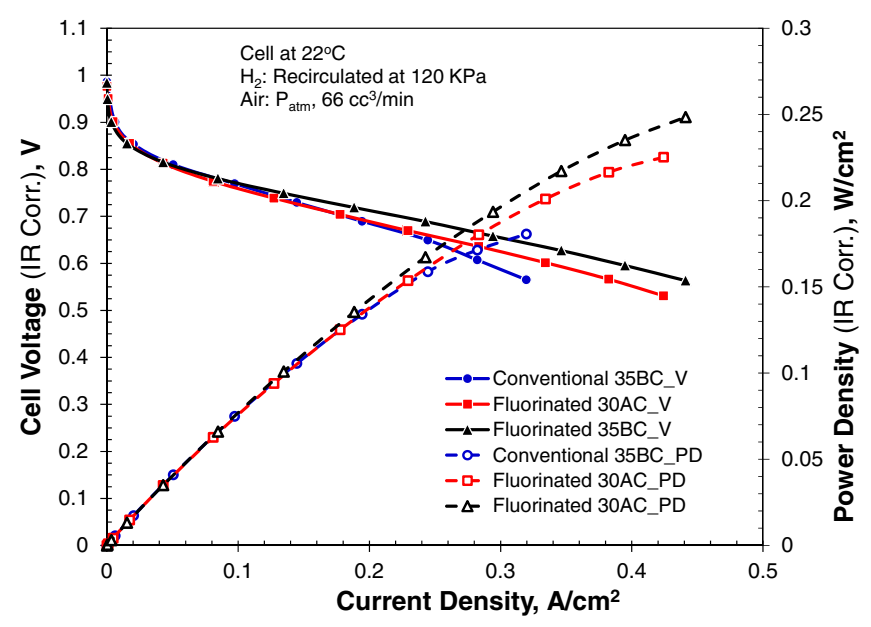

Figure 9. Fuel cell performance obtained with cathodes with conventionally wet-proofed SGL 35 BC, fluorinated SGL 30 AC, and fluorinated SGL 35 BC. $\mathrm{H}_{2}$ was continuously recirculated at $120 \mathrm{KPa}$, and air was fed at ambient pressure and a constant flow rate of $66 \mathrm{cc} / \mathrm{min}$ or $1.8 \mathrm{~A} / \mathrm{cm}^{2}$ equivalent.

Figure 8 shows the capillary pressure curves of a bilayer SGL 30 $\mathrm{AC}$ as received and after treatment by direct fluorination. An SGL 30 AC GDM has a non-wet proofed (0\% PTFE) macroporous layer and a hydrophobic MPL. During direct fluorination, both of the exposed carbon fiber surface in the macroporous layer and the exposed carbon powder in the MPL would be fluorinated. The capillary-pressure curves were obtained only for the macroporous layers of these materials. In this test, liquid water was injected into and withdrawn from the sample through the surface of the macroporous layer. The MPL, because of its higher hydrophobicity, does not significantly affect the capillary pressure curve of the macroporous layer especially in the low capillary-pressure region. ${ }^{37}$ The MPL in this case acted as a porous hydrophobic medium on top of the GDL. As observed with the single layer Toray 060 GDM in Figure 7, the directly fluorinated SGL 30 AC sample had lower $S_{\min }$ and $S_{\mathrm{Pc} 0}$ than those of the as-received non-wet proof SGL $30 \mathrm{AC}$.

Fuel cell testing. - Fluorinated SGL 35 BC was added to the study to see the effect of direct fluorination on a conventionally treated GDM. As highlighted earlier, since the SGL 35 BC GDM is already wet-proofed by the conventional method, treating it with direct fluorination would basically wet-proof the remaining uncovered carbon surface in the macroporous and MPL of this material or make it into a completely wet-proofed material. We would expect this fluorinated material to have a lower $S_{\mathrm{Pc} 0}$, higher gas permeability and, therefore, better fuel-cell performance than the conventionally treated GDM. Figure 9 shows the fuel-cell test results obtained with these materials in the same fuel cell operated at room temperature $\left(\sim 22^{\circ} \mathrm{C}\right)$, a low temperature condition where we would expect water flooding to be more severe. The data were corrected for IR loss using the resistance measured by EIS at open circuit. As shown in Figure 9, the fuel cells with the standard $35 \mathrm{BC}$ cathode and the fluorinated $30 \mathrm{AC}$ cathode gave the same performance up to around $0.2 \mathrm{~A} / \mathrm{cm}^{2}$. Beyond this current density, their performance began to deviate with the fuel cell with the conventionally treated $35 \mathrm{BC}$ GDM at the cathode showing a steeper $\mathrm{dV} / \mathrm{dI}$ slope as a result of higher concentration polarization or mass-transport losses than that of the fuel cell with the fluorinated 30 AC GDM. The fuel cell with the fluorinated 35 BC GDM at the cathode had the same performance as the other two cells up to about $0.1 \mathrm{~A} / \mathrm{cm}^{2}$, but maintained much better performance beyond this current density showing negligible mass-transport effect as shown by the relatively constant $\mathrm{dV} / \mathrm{dI}$ slope up $0.45 \mathrm{~A} / \mathrm{cm}^{2}$. This superior performance could be attributed to the fact that the SGL 35 GDM has slightly higher porosity and gas permeability than the SGL 30 GDM (see Table I), and when this SGL 35 GDM was better wet-proofed, it could 

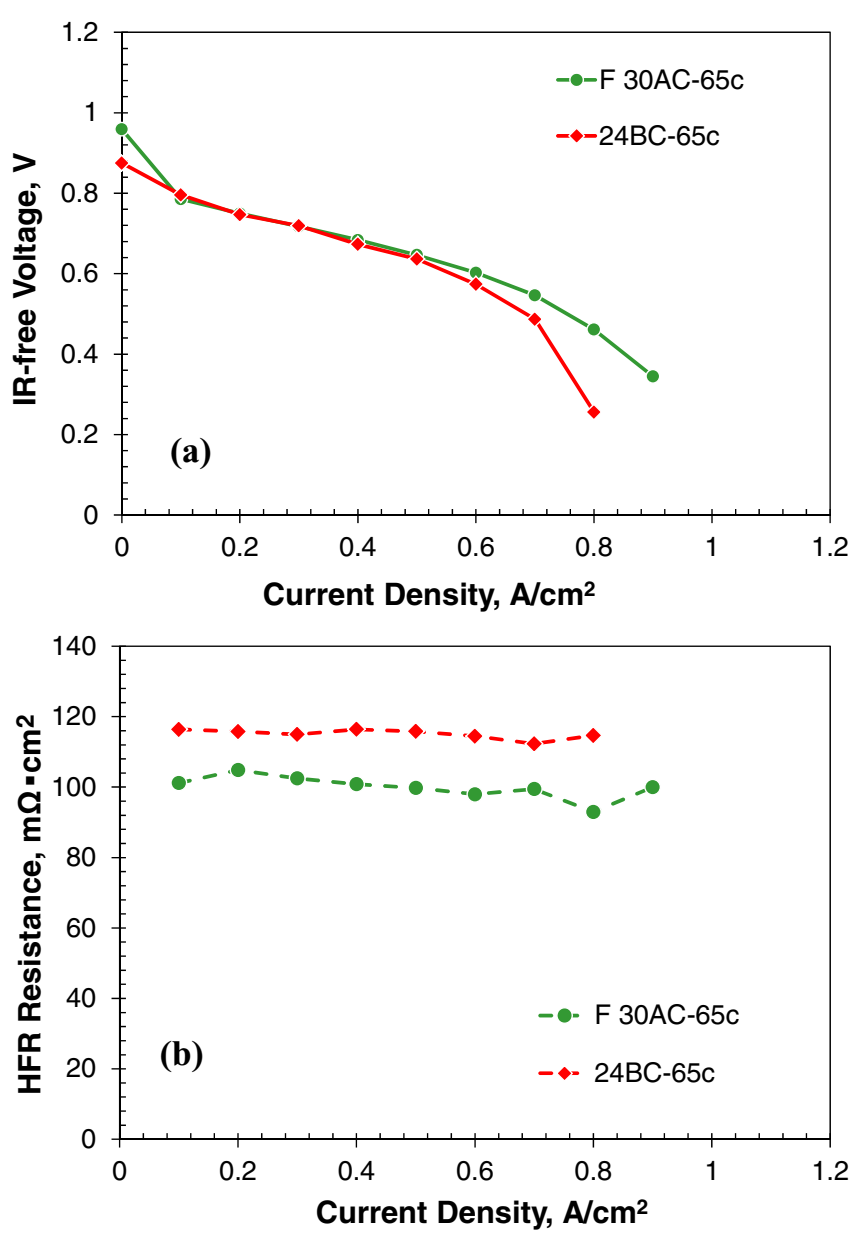

Figure 10. Fuel cell performance (a) and cell HFR internal resistance (b) obtained with cathodes made of conventional SGL 24 BC and fluorinated SGL $30 \mathrm{AC} . \mathrm{H}_{2}$ and air were fed at ambient pressure and a constant flow rate of $50 \mathrm{cc} / \mathrm{min}$ or $60 \mathrm{~A} / \mathrm{cm}^{2}$ and $25 \mathrm{~A} / \mathrm{cm}^{2}$ equivalent for $\mathrm{H}_{2}$ and air respectively.

result in better performance than the conventionally treated SGL 35 GDM where not all the carbon surface of the GDM was coated with PTFE.

Figure 10 shows the fuel-cell performance (a) and internal (HFR) ohmic resistance (b) during operation of the fuel cell tested at Tokyo Tech. This fuel cell used SGL 24 BC, a thinner $(\sim 200 \mu \mathrm{m})$ GDM that is supposed to give better performance by reducing the transport distance, for the anode and the same GDM for the cathode for the base case and a fluorinated SGL $30 \mathrm{AC}(\sim 300 \mu \mathrm{m})$ for the cathode in the other case in this comparative performance study. Note that this test was conducted at a high temperature $\left(65^{\circ} \mathrm{C}\right)$ where higher current densities could be obtained. The cell voltage presented in Figure 10 was also corrected for IR loss as in the study presented in Figure 9. The results show that the fuel cell with the cathode made of the fluorinated SGL30 AC gave better fuel-cell performance beyond $0.5 \mathrm{~A} / \mathrm{cm}^{2}$ and lower internal ohmic resistance over the whole current density range. Figure 11 shows the liquid-water distribution in these two fuel cells at $0.8 \mathrm{~A} / \mathrm{cm}^{2}$ obtained by soft X-ray radiography. These water distribution results are quite illuminating. For the fuel cell with the conventional SGL $24 \mathrm{BC}$ at the cathode we saw most the liquid water situated in the cathode GDL and gas channels. For the fuel cell with the fluorinated SGL 30 AC, we saw very little water in the cathode gas channel and most of the water in the cathode GDL was situated above the ribs of the flow field while the cathode GDL region above the flow channel was more open for gas transport. Furthermore, the fuel cell with the fluorinated GDM also showed a highly water-saturated

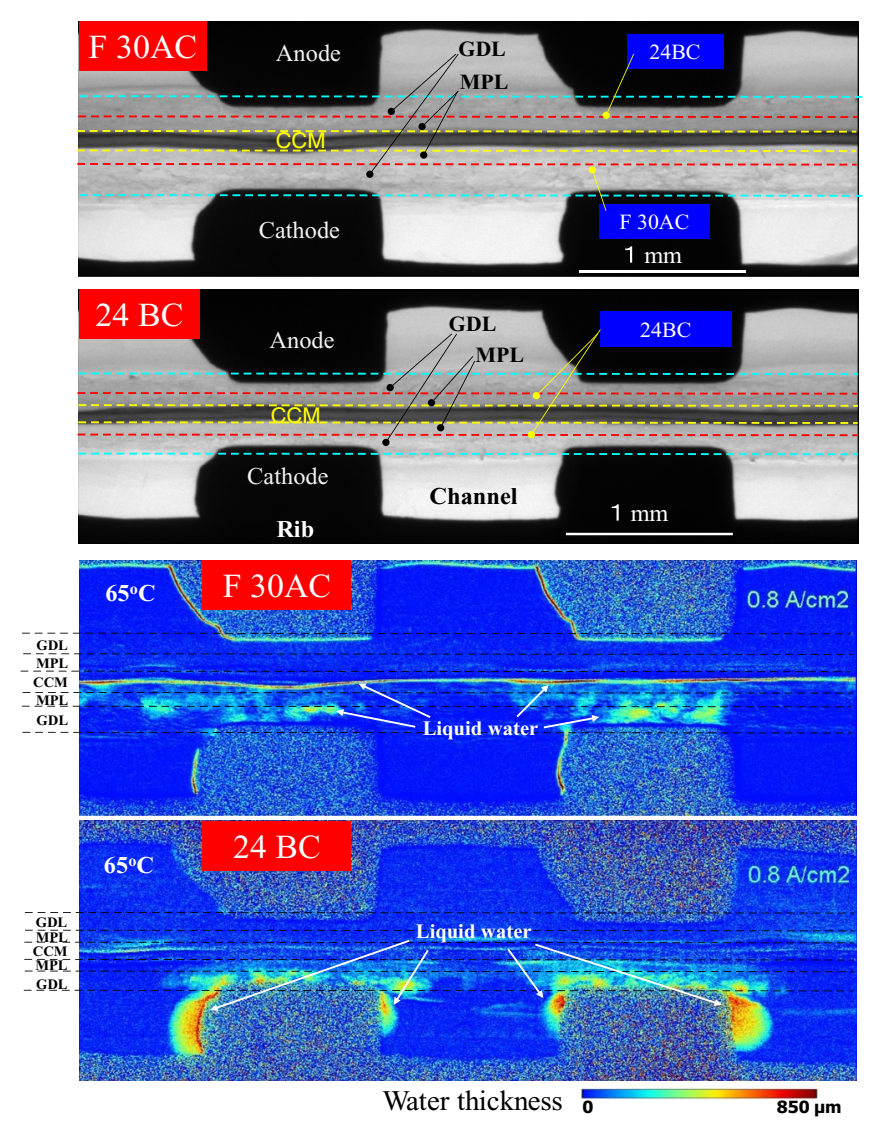

Figure 11. Liquid water distribution in fuel cells with cathodes made of conventional SGL $24 \mathrm{BC}$ and fluorinated SGL $30 \mathrm{AC}$ at $65^{\circ} \mathrm{C}$ operation obtained by soft X-ray radiography, upper figure shows the fuel cell layout and lower figure shows the water distributions.

membrane. These results support our earlier hypothesis that a highly hydrophobic GDM at the cathode would help create high capillary pressure (i.e., liquid pressure) condition at the cathode that helps drive water through the membrane back to the anode and reduce the amount of water transported out of the cathode. With less water present in the cathode GDM, a reactant gas like oxygen would have better access to the catalyst layer; and with more water transported back to the anode, one would expect the membrane to be more hydrated as observed in the fuel cell with the fluorinated GDM. This last point agrees well with the low ohmic resistance results obtained with the fuel cell with the fluorinated GDM at the cathode.

\section{Conclusions}

Direct fluorination by reacting carbon substrate with fluorine gas was found to be a suitable way to wet-proof carbon gas-diffusion media (GDM). The optimal temperature range for fluorination was found to be between $350^{\circ} \mathrm{C}$ and $400^{\circ} \mathrm{C}$. The reaction time depends on the amount of expose carbon surface that needs to be treated. For the gas-diffusion media used in this study (Toray 060 and SGL 35 and 30) 30 to 60 minutes with a $20 \%$ fluorine-argon gas mixture was sufficient.

Gas-diffusion media treated by direct fluorination had similar or slightly higher surface static water-droplet contact angles. However, the capillary-pressure properties such as liquid-water saturation at zero capillary pressure, $\mathrm{S}_{\mathrm{Pc} 0}$, and the difference between the irreducible saturation level and saturation at zero capillary pressure $\left(\mathrm{S}_{\mathrm{Pc} 0}-\mathrm{S}_{\min }\right)$ of the direct fluorinated media were lower than those of conventionally PTFE-treated media. These changes directly correlated with fuel-cell 
test results obtained at room temperature and $65^{\circ} \mathrm{C}$, which consistently showed better performance when fluorinated GDM were used at the cathode. Liquid-water distribution results obtained at a relative high current density, $0.8 \mathrm{~A} / \mathrm{cm}^{2}$, from soft X-ray radiography, showed less water content in the GDL and flow channels on the cathode side and a more hydrated membrane. These observations support the hypothesis that having a highly hydrophobic GDM at the cathode helps create a high capillary-pressure condition at the cathode that drives water back to the anode and reduces the effective liquid pressure and amount of water transported out of the cathode. With less water present in the cathode GDM, oxygen has better access to the catalyst layer, and with more water transported back to the anode, one expects the membrane to remain more hydrated. This last point agrees well with the low ohmic resistance results obtained with the fuel cell with the fluorinated GDM at the cathode.

\section{Acknowledgments}

The work done at the University of Kansas was funded by the U.S. National Science Foundation through grant number EFRI-1038234. Anthony Kwong and Adam Z. Weber were supported by the Assistant Secretary for Energy Efficiency and Renewable Energy, Fuel Cell Technologies Office, of the U.S. Department of Energy under contract number DE-AC02-05CH11231. Soft X-ray visualization techniques at Tokyo Tech were developed with the financial support of the New Energy and Industrial Technology Development Organization (NEDO) of Japan. Finally, we want to thank Regis Dowd Jr. in the KU research group for preparing the appendix.

\section{Appendix: Contact Angle Measurement}

(a) Hydrophilic Case

(b) Hydrophobic Case

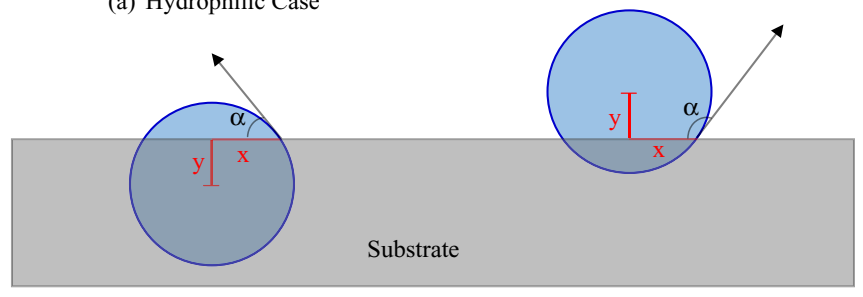

The contact-angle measurement involves the following steps:

1) Acquiring a photograph of the water droplet ( $<5$ microliters to minimize the effect of gravity) on the substrate surface with the substrate surface appearing as horizontally as possible,

2) Fitting a circle to the droplet and measuring the distances of " $y$ " and " $\mathrm{x}$ " as shown in the schematic above,

3) If the center of the circle is above the substrate, $\alpha=\tan ^{-1}(y / x)+90^{\circ}$,

4) If the center of the circle is at the substrate surface, $\alpha=90^{\circ}$, and

5) If the center of the circle is below the substrate, $\alpha=90^{\circ}-\tan ^{-1}(\mathrm{y} / \mathrm{x})$

\section{References}

1. M. Mathias, J. Roth, J. Fleming, and W. Lehnert "Diffusion Media Materials and Characterization," in Handbook of Fuel Cells - Fundamentals, Technology and Applications, W. Vielstich, H. A. Gasteiger, and A. Lamm, Eds. John Wiley \& Sons, Vol. 3: Fuel Cell Technology and Applications, 517 (2003)

2. C. Lim and C. Y. Wang, "Effects of hydrophobic polymer content in GDL on power performance of a PEM fuel cell," Electrochimica Acta, 49(24), 4149 (2004).

3. G. Lin and T. V. Nguyen, "Effect of thickness and hydrophobic polymer content of the gas diffusion layer on electrode flooding level in a PEMFC," Journal of the Electrochemical Society, 152(10), A1942 (2005).

4. J. M. Song, H. Uchida, and M. Watanabe, "Effect of wet-proofing treatment of carbon backing layer in gas diffusion electrodes on the PEFC performance," Electrochemistry, 73(3), 189 (2005).

5. Xuhai Wang and Trung Van Nguyen, "Modeling the Effects of the Micro-Porous Layer on the Net Water Transport Rate Across the Membrane in a PEM Fuel Cell," J. Electrochem. Soc., 157(4), B496 (2010).
6. Xuhai Wang and Trung Van Nguyen, "Modeling the Effects of Capillary Property of Porous Media on the Performance of the Cathode of a PEMFC," J. Electrochem Soc., 155(11), B1085 (2008).

7. Z. Qi and A. Kaufman, "Improvement of water management by a microporous sublayer for PEM fuel cells," J. Power Sources, 109, 38 (2002).

8. C. S. Kong, D.-Y. Kim, H.-K. Lee, Y.-G. Shul, and T.-H. Lee, J. Power Sources, 108 185 (2002).

9. S. Park, J.-W. Lee, and B. N. Popov, "Effect of carbon loading in microporous layer on PEM fuel cell performance," J. Power of Sources, 163, 357 (2006).

10. S. Park, J.-W. Lee, and B. N. Popov, "Effect of PTFE content in microporous layer on water management in PEM fuel cells," J. Power of Sources, 177, 857 (2008).

11. C.-J. Tseng and S.-K. Lo, "Effects of microstructure characteristics of gas diffusion layer and microporous layer on the performance of PEMFC," Energy Conversion and Management, 51, 677 (2010)

12. M. V. Williams, E. Begg, L. Bonville, H. R. Kunz, and J. M. Fenton, "Characterization of Gas Diffusion Layers for PEMFC," J. Electrochem Soc., 151(8), A1173 (2004).

13. E. Şengül, S. Erkan, I. Eroğlu, and N. Baç (2008), "Effect of gas diffusion laye characteristics and addition of pore forming agents on the performance of polymer electrolyte membrane fuel cells," Chemical Engineering Communications, 196, 161 (2008).

14. D. Cheng, S. Y. Ye, and E. Gyenge, "Reactive sensor for investigation of gas diffusion layer hydrophobicity in PEM fuel cells," Electrochemical and Solid State Letters, 11(8), B148 (2008).

15. A. Bazylak, D. Sinton, Z. Liu, and N. Djilali, "Effect of compression on liquid water transport and microstructure of PEMFC gas diffusion layers," Journal of Power Sources, 163, 784 (2007)

16. E. D. Wang, P. F. Shi, and C. Y. Du, "Treatment and characterization of gas diffusion layers by sucrose carbonization for PEMFC applications," Electrochemistry Communications 10(4), 555 (2008).

17. T. V. Nguyen, A. Ahosseini, and X. Wang, "Gas Diffusion Media for PEM Fue Cells by Direct Fluorination," ECS PRiME 2012 Meeting, Abstract 1628, Honolulu, Hawaii, Oct. 7-12, 2012; Trung Van Nguyen and Xuhai Wang, "Hydrophobized Gas Diffusion Layers and Method of Making the Same," U. S. Pat. Application No. US 2015/0024300 A1, January 22, 2015.

18. K. Lau, J. Bico, K. Teo, M. Chhowalla, G. Amaratunga, W. Milne, G. McKinley, and K. Gleason, "Superhydrophobic carbon nanotube forests," Nano Letters, 3(12), 1701 (2003).

19. N. Tsubokawa, "Carbon Black (Graft Copolymers)," in Polymeric Materials Encyclopedia," J. C. Salamone, Ed., CRC, Vol. 2, pp 941 (1996).

20. N. Tsubokawa, K. Kawatsura, and Y. Shirai, "Proceedings of the Eleventh International Conference on Composite Materials," in Surface Modification of Inorganic Ultrafine Particles by the Grafting of Polymers, Gold Coast, Queensland, Australia, pp 537 (1997).

21. E. B. Easton, Z. G. Qi, A. Kaufman, and P. G. Pickup, "Chemical modification of proton exchange membrane fuel cell catalysts with a sulfonated silane," Electrochemical and Solid State Letters, 4(5), A59 (2001)

22. Yohann R. J. Thomas, Anass Benayad, Maxime Schroder, Arnaud Morin, and Joel Pauchet, "New Method for Super Hydrophobic Treatment of Gas Diffusion Layers for Proton Exchange Membrane Fuel Cells Using Electrochemical Reduction of Diazonium Salt," ACS Appl. Mater. Interfaces, 7, 15068 (2015).

23. C. E. Bottani, A. Lamperti, L. Nobili, and P. M. Ossi, "Structure and mechanical properties of PACVD fluorinated amorphous carbon films," Thin Solid Films, 433, 149 (2003).

24. P. Ayala, M. E. H. da Costa, R. Prioli, and F. L. Freire, "Nano- and micro-scale wear of fluorinated carbon films," Surface \& Coatings Technology, 182, 335 (2004).

25. F. Chamssedine and D. Claves, "A kinetic, morphological and mechanistic approach of the fluorination of multiwall carbon nanotubes," Chemical Physics Letters, 454, 252 (2008).

26. H. Groult, T. Nakajima, L. Perrigaud, F. Warmont, and Y. Ohzawa, "Surface Modification of Carbonaceous Materials by Fluorinated Gases and Use as Anode in Lithium-Ion Battery," ESC Transactions, 3(36), 167 (2007).

27. A. Hamwi, H. Alvergnat, S. Bonnamy, and F. Beguin, "Fluorination of carbon nanotubes," Carbon, 35(6), 723 (1997).

28. H. Fujimoto, A. Mabuchi, T. Maeda, Y. Matsumura, N. Watanabe, and H. Touhara, "New Fluorine-Carbon Compound Prepared by the Direct Fluorination of Mesophase Pitch," Carbon, 30(6), 851 (1992).

29. T. Hayashi, D. Shimamoto, Y. A. Kim, H. Muramatsu, F. Okino, H. Touhara T. Shimada, Y. Miyauchi, S. Maruyama, M. Terrones, M. S. Dresselhaus, and M. Endo, "Selective optical property modification of double-walled carbon nanotubes by fluorination," ACS Nano, 2(3), 485 (2008)

30. Z. J. Li, G. D. Del Cul, W. F. Yan, C. D. Liang, and S. Dai, "Fluorinated carbon with ordered mesoporous structure," Journal of the American Chemical Society, 126(40), $12782(2004)$

31. H. Muramatsu, Y. A. Kim, T. Hayashi, M. Endo, A. Yonemoto, H. Arikai, F. Okino, and H. Touhara, "Fluorination of double-walled carbon nanotubes," Chemical Communications, $\mathbf{2 0 0 5}(15), 2002$ (2005).

32. J. T. Gostick, M. A. Ioannidis, M. W. Fowler, and M. D. Pritzker, "Direct measurement of the capillary pressure characteristics of water-air-gas diffusion layer systems for PEM fuel cells," Electrochem. Communications, 10, 1520 (2008).

33. T. V. Nguyen, G. Lin, X. Wang, and H. Ohn, "Measurement of Capillary Pressure Property of Gas Diffusion Media Used in PEM Fuel Cells," Electrochemical and Solid State Letters, 11(8), B127 (2008). 
34. P. Deevanhxay, T. Sasabe, S. Tsushima, and S. Hirai, "Effect of liquid water distribution in gas diffusion media with and without microporous layer on PEM fuel cell performance," Electrochemistry Communications, 34, 239 (2013).

35. Prodip K. Das, Adam Grippin, Anthony Kwong, and Adam Z. Weber, "Liquid-WaterDroplet Adhesion-Force Measurements on Fresh and Aged Fuel-Cell Gas-Diffusion Layers," Journal of The Electrochemical Society, 159(5), B489 (2012).
36. Anthony D. Santamaria, Prodip K. Das, James C. MacDonald, and Adam Z. Weber, "Liquid-Water Interactions with Gas-Diffusion-Layer Surfaces," J. Electrochem. Soc., 161(12), F1184 (2014).

37. Jeffrey T. Gostick, Michael W. Fowler, Marios A. Ioannidis, and Mark D. Pritzker, "Capillary pressure and hydrophilic porosity in gas diffusion layers for polymer electrolyte fuel cells," J. Power Sources, 156, 375 (2006). 